\title{
Multiple factor analysis of metachronous upper urinary tract transitional cell carcinoma after radical cystectomy
}

P. Wang, J.D. Luo, W.F. Wu, S. Wang, S.L. Cai, B.H. Shen, S.F. Shi, K.X. Wei, Z.G. Zhang and Z.D. Chen
Department of Urology, The First Affiliated Hospital, Medical College of Zhejiang University, Hangzhou, Zhejiang Province, China

\section{Correspondence}

Z.D. Chen and Z.G. Zhang

Department of Urology

The First Affiliated Hospital

Medical College of

Zhejiang University

79 Qing'chun Road

Hangzhou

Zhejiang Province, 310003

China

E-mail: zdchenzju@126.com or

zgzhangzju@126.com

Received July 31, 2006

Accepted February 2, 2007 ....................

\begin{abstract}
Transitional cell carcinoma (TCC) of the urothelium is often multifocal and subsequent tumors may occur anywhere in the urinary tract after the treatment of a primary carcinoma. Patients initially presenting a bladder cancer are at significant risk of developing metachronous tumors in the upper urinary tract (UUT). We evaluated the prognostic factors of primary invasive bladder cancer that may predict a metachronous UUT TCC after radical cystectomy. The records of 476 patients who underwent radical cystectomy for primary invasive bladder TCC from 1989 to 2001 were reviewed retrospectively. The prognostic factors of UUT TCC were determined by multivariate analysis using the COX proportional hazards regression model. KaplanMeier analysis was also used to assess the variable incidence of UUT TCC according to different risk factors. Twenty-two patients (4.6\%). developed metachronous UUT TCC. Multiplicity, prostatic urethral involvement by the bladder cancer and the associated carcinoma in situ (CIS) were significant and independent factors affecting the occurrence of metachronous UUT TCC $(\mathrm{P}=0.0425,0.0082$, and 0.0006 , respectively). These results were supported, to some extent, by analysis of the UUT TCC disease-free rate by the Kaplan-Meier method, whereby patients with prostatic urethral involvement or with associated CIS demonstrated a significantly lower metachronous UUT TCC disease-free rate than patients without prostatic urethral involvement or without associated CIS (log-rank test, $\mathrm{P}=0.0116$ and 0.0075 , respectively). Multiple tumors, prostatic urethral involvement and associated CIS were risk factors for metachronous UUT TCC, a conclusion that may be useful for designing follow-up strategies for primary invasive bladder cancer after radical cystectomy.
\end{abstract}

Key words - Bladder neoplasm - Transitional cell carcinoma - Upper urinary tract - Radical cystectomy - Prognosis 


\section{Introduction}

One of the most important characteristics of transitional cell carcinomas (TCC) of the bladder and upper urinary tract (UUT) is the high incidence of synchronous and/or metachronous occurrence. Thus, the development of UUT tumors after cystectomy has been studied by many groups for a long time, with the incidence being 2 to $9 \%$ in previous studies (1-9). These studies were performed in patients submitted to radical cystectomy because of various types of bladder cancer. In addition, the lack of guidelines regarding patient selection and the interval and duration of surveillance results in wide variations in practice (10). There are no studies assessing the prognostic factors for metachronous UUT TCC after radical cystectomy for primary invasive bladder cancer by multivariate analysis. The aim of the present study was to assess the incidence of UUT TCC following radical cystectomy for primary invasive bladder cancer, and to determine prognostic factors for primary invasive bladder cancer that may predict a UUT tumor.

\section{Patients and Methods}

The records of 476 consecutive patients submitted to radical cystectomy and urinary diversion for primary invasive bladder TCC between February 1989 and March 2001 were retrospectively reviewed. Preoperative evaluation included cystoscopy, transurethral resection of the bladder tumor and/or a bladder biopsy, urine cytology, computed tomography of the abdomen and pelvis, upper tract imaging by an intravenous urogram (IVU) or by retrograde pyelography, and a chest X-ray. Some patients underwent further metastatic evaluation, including a bone scan and chest computed tomography as clinically indicated. Surgery included pelvic lymphadenectomy in all patients and distal ureteric margins were submitted to frozen- section analysis.

Patients were followed up for at least 5 years or until death. The median follow-up was 67 months (range: 7 to 106). The follow-up evaluation consisted of a chest Xray, serum electrolytes, blood urea nitrogen, creatinine, and urine cytology every 3 months in the first and second years, and every 6 months thereafter. The UUT was monitored in all patients by IVU every 6 months during the first 3 years and then when clinically indicated. All patients had a normal UUT at IVU at the beginning of treatment.

The medical records of the patients were reviewed and analyzed for possible prognostic predictors such as the stage and grade of primary tumor, multiplicity, carcinoma in situ (CIS), presence of TCC in the prostatic urethra, presence of TCC in the distal ureter, tumor size, age, smoking, and sex. In addition to these variables, the interval between radical cystectomy and diagnosis of UUT TCC was recorded.

The metachronous UUT disease-free survival was calculated from the time of radical cystectomy to the date of UUT TCC occurrence and was analyzed by the Kaplan-Meier method. Univariate analysis was then used with a log-rank test, while multivariate analysis was used according to the Cox proportional hazards regression model to analyze predictive factors. For the latter analysis, all significant univariate variables were entered in a stepwise, forward-selection protocol. The disease-free period from the time of UUT TCC occurrence was determined by the Kaplan-Meier method, and differences among groups were compared by the logrank test. Two-sided $\mathrm{P}$ values of less than 0.05 denoted statistical significance and the hazard ratio is presented with the $95 \%$ confidence interval. Statistical analysis was performed using the SPSS statistical package.

\section{Results}

Table 1 lists the characteristics of the 476 
patients with primary invasive bladder cancer according to metachronous UUT TCC, including patient age, sex, smoking, stage, grade, tumor size, multiplicity, cytology, distal ureteral involvement, prostatic urethral involvement, and associated CIS. Patient age ranged from 18 to 83 years (median: 62 years). After radical cystectomy, UUT TCC developed in 22 of the 476 cases $(4.6 \%)$. Most of these metachronous UUT TCC $(86.4 \%)$ occurred within 3 years of cystectomy, and the mean interval from cystectomy to diagnosis of UUT TCC was 29.2 months. Among the primary invasive bladder cancer patients with metachronous UUT TCC after cystectomy, 17 had multifocality, 8 had prostatic urethral involvement, and 11 had associated CIS.

Eleven clinical and pathologic parameters were subjected to univariate analysis to determine whether they were statistically significant predictors of UUT TCC occurrence. In the analysis, tumor stage and grade were reclassified into two subgroups: early stage $\left(T_{2}\right)$ versus advanced stage $\left(T_{3}, T_{4}\right)$, and low grade $\left(\mathrm{G}_{1}, \mathrm{G}_{2}\right)$ versus high grade $\left(G_{3}\right)$. The following variable were of prognostic significance for all patients in the univariate analysis (Table 1$)$ : age $(\mathrm{P}=$ $0.0385)$, stage $(\mathrm{P}=0.0426)$, multiplicity $(\mathrm{P}=$ $0.0268)$, prostatic urethral involvement $(\mathrm{P}=$ $0.0075)$, and associated CIS ( $\mathrm{P}=0.0012)$.

The factors that were significant in univariate analysis were then analyzed by multivariate analysis using the COX proportional model (Table 2). Multiplicity, prostatic urethral involvement, and associated CIS were identified as being significant independent prognostic factors of metachronous UUT TCC $(\mathrm{P}=0.0425,0.0082$, and 0.0006 , respectively). The results were supported to some extent by analysis of the UUT TCC disease-free rate by the KaplanMeier method, whereby patients with prostatic urethral involvement or with associated CIS demonstrated a significantly lower metachronous UUT TCC disease-free rate than
Table 1. Clinical characteristics of the patients and univariate analysis.

\begin{tabular}{|c|c|c|c|}
\hline & Patients & UUT TCC & $P$ value \\
\hline Age (years) & & & 0.0385 \\
\hline$\geq 60$ & $286(60.8 \%)$ & $16(72.7 \%)$ & \\
\hline$<60$ & $190(39.2 \%)$ & $6(27.3 \%)$ & \\
\hline Sex & & & 0.5842 \\
\hline Men & $394(82.8 \%)$ & $17(77.3 \%)$ & \\
\hline Women & $82(17.2 \%)$ & $5(22.7 \%)$ & \\
\hline Smoking habit & & & 0.0768 \\
\hline Yes & $233(49.9 \%)$ & $14(63.6 \%)$ & \\
\hline No & $243(51.1 \%)$ & $8(36.4 \%)$ & \\
\hline Stage & & & 0.0426 \\
\hline $\mathrm{T}_{2}$ & $94(19.7 \%)$ & $5(22.7 \%)$ & \\
\hline $\mathrm{T}_{3}$ & $235(49.4 \%)$ & $13(59.1 \%)$ & \\
\hline $\mathrm{T}_{4}$ & $147(30.9 \%)$ & $4(18.2 \%)$ & \\
\hline Grade & & & 0.0832 \\
\hline $\mathrm{G}_{1}$ & $9(1.9 \%)$ & $0(0 \%)$ & \\
\hline $\mathrm{G}_{2}$ & $197(41.4 \%)$ & $9(40.9 \%)$ & \\
\hline $\mathrm{G}_{3}$ & $270(56.7 \%)$ & $13(59.1 \%)$ & \\
\hline Tumor size & & & 0.3972 \\
\hline$\geq 2 \mathrm{~cm}$ & $312(65.5 \%)$ & $12(54.5 \%)$ & \\
\hline$<2 \mathrm{~cm}$ & $164(34.5 \%)$ & $10(45.5 \%)$ & \\
\hline Multiplicity & & & 0.0268 \\
\hline Multiple & $184(38.7 \%)$ & $17(77.3 \%)$ & \\
\hline Single & $292(61.3 \%)$ & $5(22.7 \%)$ & \\
\hline Cytology & & & 0.8934 \\
\hline Positive & $315(66.2 \%)$ & $13(59.1 \%)$ & \\
\hline Negative & $161(33.8 \%)$ & 9 (40.9\%) & \\
\hline Distal ureteral involvement & & & 0.6855 \\
\hline Yes & $54(11.3 \%)$ & $3(13.6 \%)$ & \\
\hline No & $422(88.7 \%)$ & 19 (86.4\%) & \\
\hline Prostatic urethral involvement & & & 0.0075 \\
\hline Yes & $42(10.7 \%)$ & $8(47.1 \%)$ & \\
\hline No & $352(89.3 \%)$ & $9(52.9 \%)$ & \\
\hline Associated carcinoma in situ & & & 0.0012 \\
\hline Yes & $56(11.8 \%)$ & $11(50.0 \%)$ & \\
\hline No & $420(88.2 \%)$ & $11(50.0 \%)$ & \\
\hline
\end{tabular}

Data are reported as number with percent in parentheses. UUT = upper urinary tract; $T C C=$ transitional cell carcinoma; $T_{2}=$ early stage; $T_{3}, T_{4}=$ advanced stages; $G_{1}, G_{2}$ = low grades; $\mathrm{G}_{3}=$ high grade .

Table 2. Cox proportional hazards model analysis of predictors of metachronous upper urinary tract transitional cell carcinoma due to primary invasive bladder cancer.

\begin{tabular}{lcc}
\hline Predictors & P value & Hazard ratio (95\%Cl) \\
\hline $\begin{array}{l}\text { Age, } \geq 60 \text { vs }<60 \text { years } \\
\text { Stage }\end{array}$ & 0.1258 & $1.41(1.04-1.92)$ \\
$\quad$ Stage 2 vs stage 3 & 0.0632 & $0.83(0.57-1.15)$ \\
$\quad$ Stage 2 vs stage 4 & 0.0843 & $1.79(1.17-2.74)$ \\
Multiplicity, multiple vs single & 0.0425 & $2.98(2.02-3.56)$ \\
Prostatic urethral involvement, yes vs no & 0.0082 & $2.76(1.86-4.19)$ \\
Associated carcinoma in situ, yes vs no & 0.0006 & $4.32(2.15-6.68)$ \\
\hline
\end{tabular}


patients without prostatic urethral involvement or without associated CIS ( $\mathrm{P}=0.0116$ and 0.0075, respectively; Figures 1 and 2). Multiplicity also affected the disease-free rate for metachronous UUT TCC. Although not significant, the UUT TCC disease-free rate of single focus disease tended to be higher than that of multiple focus disease ( $\mathrm{P}$ $=0.0895$ by the log-rank test).

\section{Discussion}

Patients who survive bladder cancer remain at risk for upper tract recurrence due to the multifocal nature of urothelial cancer. Approximately 2 to $9 \%$ of patients with bladder cancer develop metachronous UUT TCC, usually within 3 years of cystectomy for bladder cancer $(1-7,9,10)$. In our series of primary invasive bladder cancer, the rate of metachronous UUT TCC was 4.6\% (22 patients), and 19 of these patients $(86.4 \%)$ developed UUT TCC within 3 years of radical cystectomy. The incidence and high risk period were similar to those reported previously $(1-9,11,12)$.

Several studies have assessed the influence of bladder CIS on the incidence of

Figure 1. Kaplan-Meier plot for freedom from metachronous upper urinary tract (UUT) transitional cell carcinoma (TCC) comparing groups with and without prostatic urethral involvement $(P=0.0116$, log-rank test $)$.

Figure 2. Kaplan-Meier plot for freedom from metachronous upper urinary tract (UUT) transitional cell carcinoma (TCC) comparing groups with and without associated carcinoma in situ ( $P=0.0075$, log-rank test).
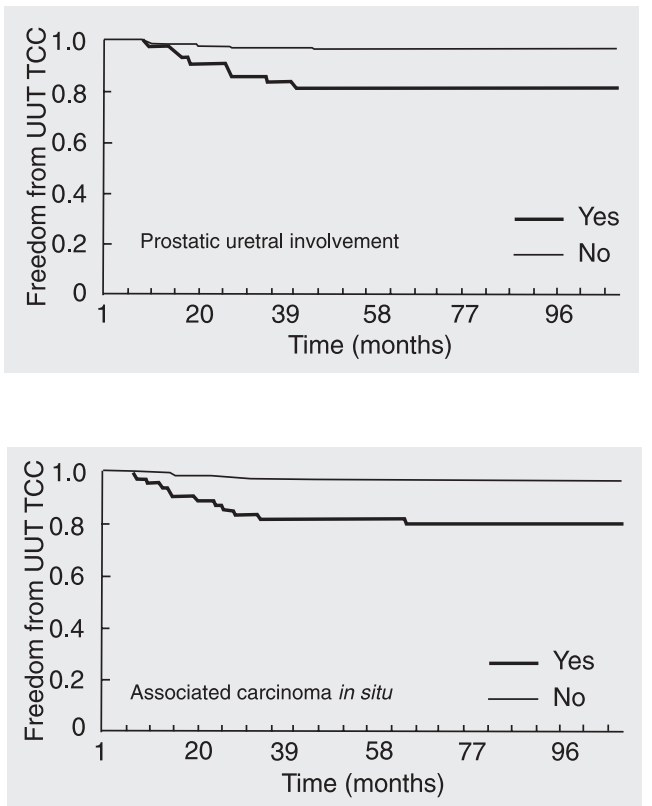

upper-tract recurrence. Zincke et al. (1) reported that 8 of 14 patients with an upper tract tumor after cystectomy had CIS in the cystectomy specimen. In that series, patients with CIS were three times more likely to develop a UUT TCC. A similar but not significant rate was reported by Balaji et al. (11). However, Solsona et al. (13) reported that in patients undergoing cystectomy the incidence of UUT TCC was higher among cases with bladder CIS ( 8 of $46,17.4 \%$ ) than among cases with invasive bladder cancer (7 of $179,3.9 \%)(\mathrm{P}<0.01)$. Using Cox's proportional hazards regression model (Table 2), we compared metachronous UUT TCC recurrence rates after primary invasive bladder cancer with those after associated CIS and identified a higher incidence after associated CIS. The incidence of UUT TCC recurrence was 11 of $56(19.6 \%)$ for associated CIS compared with 11 of $420(2.6 \%)$ for invasive bladder cancer. A similar result was obtained by Kaplan-Meier analysis (Figure $2, \mathrm{P}=0.0075)$. Freedom from UUT TCC was better for patients with bladder cancer if they did not have an associated CIS. Our results demonstrate that associated CIS was a powerful predictor for metachronous UUT TCC after primary invasive bladder cancer. Based on the fact that different studies report different statistical results, we think that this discrepancy might be attributed to follow-up bias because the absolute incidence in any given series depends on the duration of follow-up as well as the degree of surveillance.

Although prostatic urethral involvement is widely accepted as a risk factor for upper tract recurrence, controversy exists regarding whether there is a statistically significant association between TCC involving the prostate and the eventual development of a UUT TCC after radical cystectomy. Kenworthy et al. (6) reported that TCC within the prostate was present in 101 of 334 men (30\%) who underwent cystectomy. Five of these patients $(5.0 \%)$ had UUT TCC compared to 4 of $232(1.8 \%)$ without TCC of the prostate, 
with no statistically significant difference. In contrast, Sved et al. (14) observed TCC within the prostatic urethra in 27 of 235 (11\%) patients. Of the 5 patients with eventual UUT TCC, 3 had TCC involving the prostatic urethra compared with 24 of 230 (10\%) patients with no UUT TCC. The relationship between prostatic urethral TCC and eventual UUT TCC was statistically significant $(\mathrm{P}<0.01)$ and multivariate analysis showed that the presence of prostatic urethral TCC was an independent predictor of UUT TCC. Nevertheless, our results agreed with the conclusion of Sved et al. (14) by multivariate analysis using the Cox proportional hazards model (Table 2, $\mathrm{P}=0.0082$ ) and analysis by the Kaplan-Meier method for freedom from UUT TCC (Figure 1, P = 0.0116), although there were some differences in the characteristics of the patients studied. Considering these data as a whole, we believe that statistical contradiction in different series might be attributed to the following facts: a) because there was some correlation between the statistical result and the number of samples, a much larger study population would be necessary to demonstrate the significance of this relationship; $b$ ) some studies did not differentiate between cases with direct involvement of prostate stroma by the bladder tumor and non-contiguous TCC involving the prostatic urethra. The true incidence of eventual UUT in patients with TCC involving the prostatic urethra is unknown. A possible explanation for the lack of association between prostatic urethral involvement and metachronous UUT TCC is that the authors did not distinguish between non-contiguous prostatic urethral involvement and direct prostate invasion by bladder TCC (14).

Other proposed risk factors for UUT TCC after radical cystectomy such as age, stage, and multiplicity were analyzed by multivariate analysis. We observed that multiplicity was the only prognostic predictor $(\mathrm{P}=0.0425$, hazard ratio $2.98,95 \%$ confidence interval $=$
2.02-3.56), although the result was not supported by analysis of freedom from UUT TCC by the Kaplan-Meier method ( $\mathrm{P}=$ 0.0895). Thus, the risk of metachronous UUT TCC is 3-fold higher for multiple than for single bladder tumors. Previous studies have demonstrated that multiplicity is a risk factor for UUT TCC after the diagnosis of superficial bladder cancer $(15,16)$. Of particular interest is our finding that there is a similar correlation between multifocal primary invasive bladder and metachronous UUT TCC after radical cystectomy. Different pathogenetic mechanisms have been proposed. First, the entire urothelium from the renal pelvis to the urethra may be susceptible to malignant transformation due to a carcinogenic insult. Second, several clinical and experimental findings support the alternative explanation that the multifocal development of urothelial cancers is due to seeding and/or intraepithelial spreading of the original cancer cells $(17,18)$. Furthermore, detailed investigations of multifocal urothelial cancers, including superficial disease, have suggested the development of multifocal cancer from plural clones $(19,20)$. However, the precise mechanism of multifocal development in urothelial cancers requires further elucidation.

Tumor multiplicity, associated CIS and prostatic urethral involvement all had a significant impact on the risk of metachronous UUT TCC for primary invasive bladder cancer after radical cystectomy. We noted that the risk of the development of UUT TCC after excision of primary bladder cancer varied according to different predictors, which may be useful for designing strategies to follow these cases.

\section{Acknowledgments}

We thank Dr. Cheng-Tan Li (Department of Pharmacology, Medical College of Zhejiang University) for help with the statistical analysis. 


\section{References}

1. Zincke H, Garbeff PJ, Beahrs JR. Upper urinary tract transitional cell cancer after radical cystectomy for bladder cancer. J Urol 1984; 131 : 50-52.

2. Hastie KJ, Hamdy FC, Collins MC, Williams JL. Upper tract tumours following cystectomy for bladder cancer. Is routine intravenous urography worthwhile? $\mathrm{Br} J$ Urol 1991; 67: 29-31.

3. Tsuji $Y$, Nakamura $H$, Ariyoshi A. Upper urinary tract involvement after cystectomy and ileal conduit diversion for primary bladder carcinoma. Eur Urol 1996; 29: 216-220.

4. Mufti GR, Gove JR, Riddle PR. Nephroureterectomy after radical cystectomy. J Urol 1988; 139: 588-589.

5. Malkowicz SB, Skinner DG. Development of upper tract carcinoma after cystectomy for bladder carcinoma. Urology 1990; 36: 20-22.

6. Kenworthy P, Tanguay S, Dinney CP. The risk of upper tract recurrence following cystectomy in patients with transitional cell carcinoma involving the distal ureter. J Urol 1996; 155: 501-503.

7. Huguet-Perez J, Palou J, Millan-Rodriguez F, Salvador-Bayarri J, Villavicencio-Mavrich $\mathrm{H}$, Vicente-Rodriguez J. Upper tract transitional cell carcinoma following cystectomy for bladder cancer. Eur Urol 2001; 40: 318-323.

8. Kirkali Z, Tuzel E. Transitional cell carcinoma of the ureter and renal pelvis. Crit Rev Oncol Hematol 2003; 47: 155-169.

9. Holmang S, Johansson SL. Bilateral metachronous ureteral and renal pelvic carcinomas: incidence, clinical presentation, histopathology, treatment and outcome. J Urol 2006; 175: 69-72.

10. Enver MK, Miller PD, Chinegwundoh FI. Upper tract surveillance in primary bladder cancer follow-up. BJU Int 2004; 94: 790-792.

11. Balaji KC, McGuire M, Grotas J, Grimaldi G, Russo P. Upper tract recurrences following radical cystectomy: an analysis of prognostic factors, recurrence pattern and stage at presentation. J Urol 1999;
162: 1603-1606.

12. Huang PC, Huang CY, Huang SW, Lai MK, Yu HJ, Chen J, et al. High incidence of and risk factors for metachronous bilateral upper tract urothelial carcinoma in Taiwan. Int J Urol 2006; 13: 864-869.

13. Solsona E, Iborra I, Ricos JV, Dumont R, Casanova JL, Calabuig C. Upper urinary tract involvement in patients with bladder carcinoma in situ (Tis): its impact on management. Urology 1997; 49: 347-352.

14. Sved PD, Gomez P, Nieder AM, Manoharan M, Kim SS, Soloway MS. Upper tract tumour after radical cystectomy for transitional cell carcinoma of the bladder: incidence and risk factors. BJU Int 2004; 94: 785-789.

15. Palou J, Farina LA, Villavicencio H, Vicente J. Upper tract urothelial tumor after transurethral resection for bladder tumor. Eur Urol 1992; 21: 110-114.

16. Millan-Rodriguez F, Chechile-Toniolo G, Salvador-Bayarri J, HuguetPerez J, Vicente-Rodriguez J. Upper urinary tract tumors after primary superficial bladder tumors: prognostic factors and risk groups. J Urol 2000; 164: 1183-1187.

17. Xu X, Stower MJ, Reid IN, Garner RC, Burns PA. Molecular screening of multifocal transitional cell carcinoma of the bladder using p53 mutations as biomarkers. Clin Cancer Res 1996; 2: 1795-1800.

18. Habuchi T, Takahashi R, Yamada H, Kakehi Y, Sugiyama T, Yoshida O. Metachronous multifocal development of urothelial cancers by intraluminal seeding. Lancet 1993; 342: 1087-1088.

19. Harris $A L$, Neal DE. Bladder cancer - field versus clonal origin. $N$ Engl J Med 1992; 326: 759-761.

20. Takahashi T, Habuchi T, Kakehi Y, Mitsumori K, Akao T, Terachi T, et al. Clonal and chronological genetic analysis of multifocal cancers of the bladder and upper urinary tract. Cancer Res 1998; 58: 5835-5841. 